\title{
An appraisal of various pathogen detection methods in eggs and poultry
}

\author{
D A. A. P. Milton ${ }^{a}$, (D) G. Bhuvana Priya ${ }^{b}$, K. M. Momin ${ }^{c}$, M. Angappan ${ }^{\text {, }}$ \\ Damir Das ${ }^{\mathrm{a}}$, (D) S. Ghatak \\ a Scientist, Division of Animal Health, ICAR Research Complex for NEH Region, Umiam, Meghalaya \\ ${ }^{b}$ Assistant Professor, College of Agriculture, Central Agricultural University, Kyrdemkulai, Meghalaya \\ ${ }^{c}$ Project Assistant, College of Agriculture, Central Agricultural University, Kyrdemkulai, Meghalaya

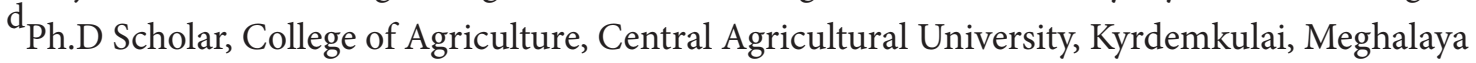

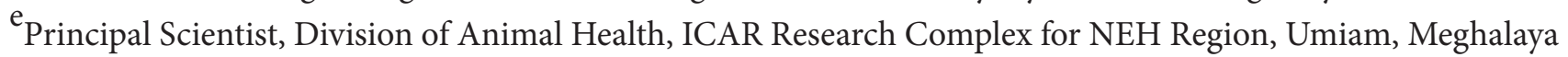

Corresponding author: A. A.P. Milton | email: vetmilton@gmail.com

Co-author: GBV: bhuvana.priya20@gmail.com, KMM: kasnchi95@gmail.com, MA: angappandr@gmail.com, SD: drsamirvph@yahoo.com, SG: ghataksnd@rediffmail.com

Received: 09-10-2020, Accepted: 21-11-2020, Published online: 21-12-2020

\begin{abstract}
To ensure safety in egg and poultry products, timely detection of pathogenic microbes is of paramount importance. This review offers an appraisal of different routinely used and novel emerging pathogen detection methods in egg, poultry and their products. Timely detection of pathogens is decisive to curtail outbreak risks, reduce hospitalisation, and provide product assurance. It will also reduce the cost of holding food products in cold storage and reduces product recalls. Some crucial issues need to be taken care of in choosing or developing a foodborne pathogen detection method. They are requirement of costly or sophisticated equipment, portability, trained personnel, viable but non-culturable bacteria (may give false-negative results), dead microbes (may give falsepositive results), stressed or sub-lethally damaged bacteria and slow-growing microbes (require enrichment). In this review, the focus has been given on culture-based methods, nucleic acid-based methods, immunological methods and biosensor based methods.
\end{abstract}

Keywords: Egg; poultry; detection methods; product assurance; safety.

doi: https://doi.org/10.51128/jfas.2020.A017 | How to cite this article: Milton, A. A. P., Priya, G.B., Momin, K. M., Angappan, M., Das, S. and Ghatak, S. 2020. An appraisal of various pathogen detection methods in eggs and poultry. Journal of Food and Animal Sciences, 01(02): 93-97. Copyright: Milton et al., 2020. Open Access. This article is distributed under the terms of the Creative Commons Attribution 4.0 International License (http://creativecommons.org/licenses/by/4.0/), which permits unrestricted use, distribution, and reproduction in any medium, provided you give appropriate credit to the original author(s) and the source, provide a link to the Creative Commons license, and indicate if changes were made. The Creative Commons Public Domain Dedication waiver (http://creativecommons.org/publicdomain/zero/1.0/) applies to the data made available in this article, unless otherwise stated. 


\section{Introduction}

Egg and poultry are regarded as a food of high-level nutritive value and gaining popularity and preference among consumers throughout the world as it can offer high-quality proteins, minerals, vitamins, and other nutrients to improve and sustain human health. To meet the higher necessities of consumers and keep the market competitive, the food industry should produce not only quality stuff but also safer stuff. As far as egg/poultry-based foods are concerned, there are glut of potential microbial hazards like Salmonella, Campylobacter, Listeria monocytogenes, etc. In each year, in the USA alone, 48 million illnesses and 3000 deaths have been attributed to foodborne illnesses, resulting in US\$78 billion economic expenses. Particularly, eggs and poultry were associated with $19 \%$ deaths, with most deaths being attributed to Salmonella and L. monocytogenes (Bhunia, 2014). Broiler meat is the single key source of human campylobacteriosis (Kaakoush et al., 2015). Animal origin food safety is a complex issue facing a number of challenges like traceability issues, regulatory issues, pathogen and residue detection hitches, antibiotic resistance, other consumer concerns, etc., (Bhunia, 2014). Therefore detection of pathogenic microbes in egg and poultry products is essential to ensure safety. It is also essential to detect early to reduce untoward effects on consumer health and food business. Apart from curtailing the risk of outbreaks it also provides product assurance. Detection and identification of the pathogen are also vital to develop an intervention plan to reduce or eliminate the pathogen (Wang and Salazar, 2016). In the present discourse, the focus has been provided to the comprehensive discussion and appraisal of conventional culture-, immunological-, molecular-, and biosensor-based techniques (Figure $1)$.

\section{Intricacies of pathogen detection methodologies}

To evaluate a detection method, two performance criteria are often used i.e., assay accuracy (lower limit of detection as low as one target organism per 25 to $325 \mathrm{~g}$ of sample) and time to result (food sample to the final result). A tool that gives more false-negative results is more detrimental than the one which gives more false-positive results, as the former is the biggest concern for outbreaks due to distribution of pathogen contaminated foods. The time taken for giving final results may vary from hours to days. A technology taking lesser time with good accuracy rate is the need of the hour for the food industry as the test product could be promptly released in time (Ghatak, 2020). Since most of the test food products are turning to be negative, it would be wise to deploy a detection method that finds out negative samples rapidly, ideally in hours, so as to execute lengthy analysis (may take 2-3 days) of positive samples to isolate and type the pathogens (Bhunia, 2014). The complexity of various food matrices like the egg/meat of different poultry species, salt and fat, preservatives, cooked foods, background microbes may also stipulate the selection of detection methods (Bhunia, 2014; Priyanka et al., 2016).

\section{Traditional culture-based methods}

Culture-based methods to detect egg/poultry transmitted pathogens are widely accepted and gold standard methods. A common procedure of cultural isolation involves homogenizing or stomaching a food (egg/poultry) sample, enriching in non-selective or selective enrichment media and then streaking on selective solid agar plates followed by phenotyping to ensure the presence and identify the targets. They have legal standing as they are sensitive, accurate, dependable, and reproducible. An extensive range of specific growth media has been developed to selectively isolate the target pathogen, although general sanitation assessment methods (total coliform count, aerobic plate count) are also widely used for quantitative assessment of overall sanitary feature of egg and poultry products (Priyanka et al., 2016; Ghatak, 2020). The foremost drawback of cultural methods is its time consuming nature, taking up to 3-7 days to give negative results and for positive results, an additional 2-3 days or more may be needed. This may lead to fatalities, distribution of contaminated meat products and unnecessary accumulation of products. Another important disadvantage is the inability to detect VBNC state of bacteria, which are prevalent among food pathogens than hitherto thought. Some of the egg/poultry borne pathogens that are classified as VBNC type include $C$. jejuni, E. coli, Salmonella spp., Shigella spp. From the food safety perspective, these traditional methods are becoming less attractive as the modern food industry desires more rapid detection methods (Bhunia, 2014; Priyanka et al., 2016; Wang and Salazar, 2016). 


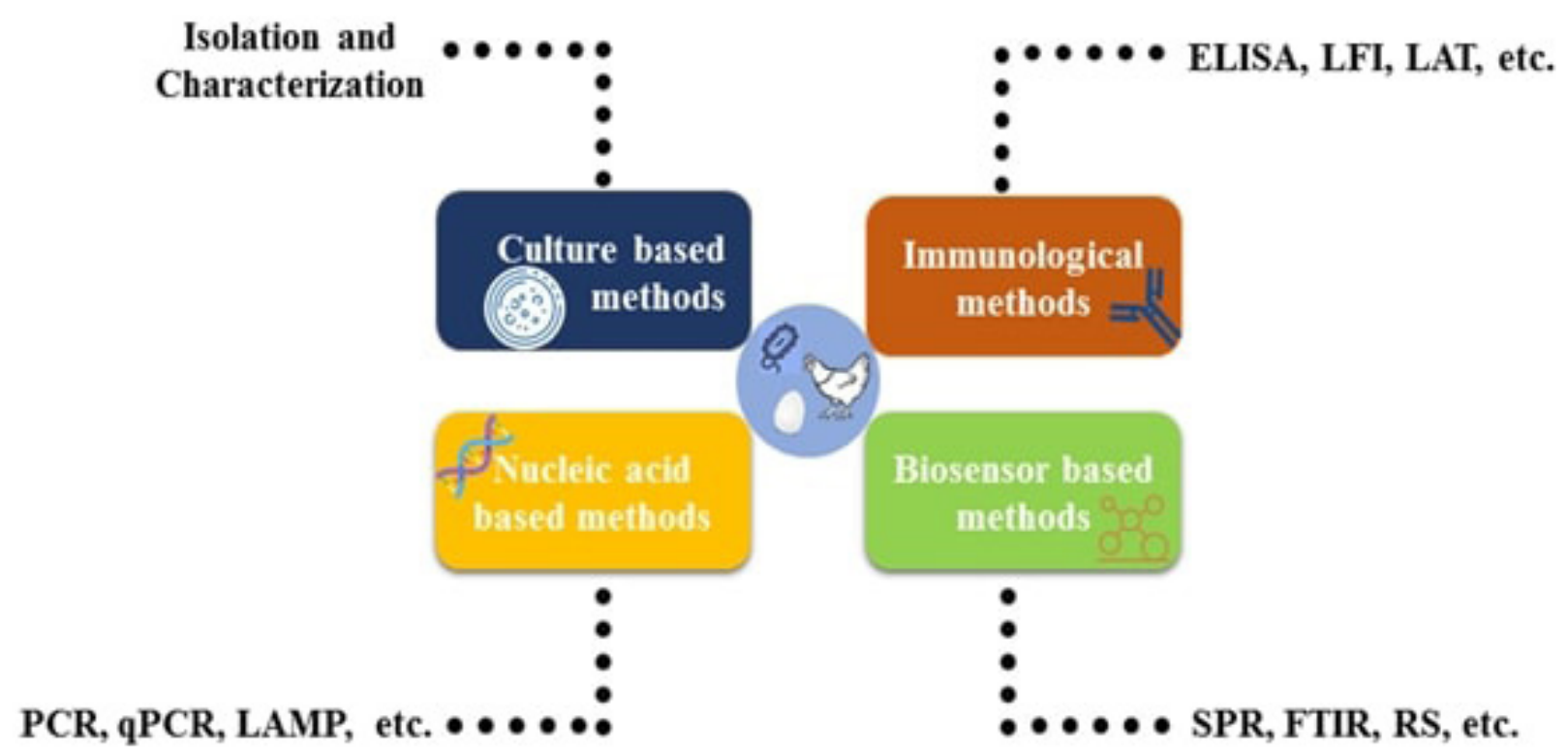

Fig.1. Common detection platforms deployed for the detection of pathogens in eggs and poultry

\section{Immunological methods}

By exploiting the ability of antigen-antibody interaction, various detection and concentration methods were developed for rapid use in the food industry. Cost-effectiveness, simple way of execution, and rapidity have made the immunological assays popular in the food industry. The critical factor of the immunological assays is the antibodies as they determine the specificity and sensitivity of any assay (Priyanka et al., 2016; Mangal et al., 2016). The limit of detection for immunological assays is roughly $10^{4}-10^{5}$ $\mathrm{CFU} / \mathrm{g}$ of food. Immuno-magnetic separation method is a sample concentration method which uses the antibodies coupled with the magnetic particles to pick up specificantigen (bacteria) from the food samples. The pathogen concentrated samples can then be detected by any of the immunological or molecular detection methods (Mangal et al., 2016). The three important categories of immunological methods deployed to screen foodborne pathogens and commercially popular are enzyme-linked immunosorbent assay (ELISA), lateral flow immunoassay (LFI) and the latex agglutination test (LAT) (Bhunia et al., 2014; Ghatak, 2020). The disadvantage of ELISA is the requirement of pure and specific antibodies, the lower limit of detection, perishable nature of reagents, need for investment costs like specialized ELISA readers. The main limitation of LFI and LAT is that they require high pathogen concentration (in a range of $10^{7}-10^{9}$ ) for a positive reaction. Drawbacks of LAT are the shorter shelf life of beads and qualitative nature (Mangal et al.,
2016).

\section{Nucleic acid-based methods}

Nucleic acid-based detection methods function by targeting specific DNA/ RNA sequences of the targeted pathogens. The advantages of nucleic acid-based methods are their higher specificity and sensitivity, less labour intensiveness, timeeffectiveness, less human errors and easy gel- or visual-based or real-time interpretation. Various formats of nucleic acid-based methods are polymerase chain reaction (PCR), multiplex PCR, viability PCR, real-time PCR, reverse transcriptase (RT) PCR, DNA microarray and isothermal-based methods like LAMP, PSR etc,. The important drawbacks of the techniques are the requirement of nucleic acid extraction, costly equipment, post PCR processing like gel electrophoresis and lack of ability for discriminative detection of live or viable pathogens steering to false positive results (Momin et al., 2020). To achieve selective detection of viable cells, certain dyes like ethidium monoazide (EMA) or propidium monoazide (PMA) have been used with PCR, which is termed as viability PCR (Pan and Breidt, 2007). RTqPCR is the preferred method of choice for screening foodborne viruses in food. Isothermal based detection techniques can be visually interpreted, they obviate the necessity of thermal cycler and gel electrophoresis, thus making them rapid and simple (Momin et al., 2020). Many commercial LAMP-based kits are available for detecting egg/poultry borne pathogens 
like Salmonella, Campylobacter, Listeria, etc (Mangal et al., 2016). The limitations of LAMP-based techniques are multiple primer requirement and carry-over or leftover contamination leading to false-positive results (Momin et al., 2020).

\section{Biosensor based methods}

Biosensors are analytical devices which alters a biological response into an electrical signal. There are two major components of any biosensors: a bioreceptor to recognize or sense the target analyte and a transducer to convert recognition into a quantifiable electrical signal. Hence, classification of biosensors can be done based on the kind of biocomponent used, mode or mechanism of signal transduction, etc., (Velusamy et al., 2010; Singh et al., 2016). The transduction elements are generally optical, piezoelectric, electrochemical and magnetoelastic and the recognition elements that are responsible for specificity can be enzymes, cell receptors, antigens, antibodies, aptamers, bacteriophages, nucleic acid probes and antimicrobial peptides (Bhunia, 2014). Biosensors are sensitive, rapid, cost-effective, require minimal sample preparation and fit for on-site detection. A combination of brief enrichment step or concentration by means of paramagnetic beads can improve the sensitivity and can offer results in 4 to 6 hours (Bhunia, 2014). Biosensors that are categorized into optical, electrical, optochemical, electrochemical, piezoelectric, magnetoelastic and mass based sensors demonstrate utility in the food industry (Bhunia, 2014). Among the optical based sensors, evanescent wave sensors, surface plasmon resonance (SPR) sensors, light-scattering sensors, Raman spectroscopy (RS), Fourier transformed infrared (FTIR) sensors and hyperspectral imaging sensors are good in producing real-time or near real-time results (Bhunia, 2014). Nanotechnology has also been coupled with biosensors as it has special properties that can facilitate detection (Wang and Salazar, 2016). Developments in new recognition methods, new transducing materials, multiplex detection configurations, wireless devices, integrated biosensing systems and microfluidic techniques would uphold biosensors to turn out to be a mature and method of choice for application in the growing poultry and food industry (Wang and Salazar, 2016).

\section{Other detection platforms}

In addition to the described detection methods, some other platforms are also available including nextgeneration sequencing (NGS) based platforms, Matrixassisted laser desorption ionization-time of flight mass spectrometry (MALDI-TOF-MS), automated microbial identification systems like VITEK, Phoenix 100 , Sensititre etc.

\section{Conclusions}

Several innovative detection methods are in the process of development emphasizing reduction of detection time, culture-free detection, and enhancement of sensitivity and limit of detection. It is more important to develop simple equipmentfree detection systems suitable for less-advanced food testing laboratories in the developing and underdeveloped nations.

\section{Authorship contribution statement}

A.A.P. Milton: Conceptualization, Writing - original draft, Writing - review \& editing; G. Bhuvana Priya: Conceptualization, Writing - original draft, Writing - review \& editing; K.M. Momin: Writing - original draft; M. Angappan: Writing - original draft; S. Das: Writing - original draft; S. Ghatak: Writing - original draft; Writing - review \& editing

\section{Acknowledgments}

All the authors acknowledge and thank their respective Institutes and Universities.

\section{Funding}

This compilation is a review article written by its authors and required no substantial funding to be stated.

\section{Declaration of Competing Interest}

All authors declare that there exist no commercial or financial relationships that could, in any way, lead to a potential conflict of interest.

\section{References}

Bhunia, A.K. 2014. One day to one hour: how quickly can foodborne pathogens be detected? Future Microbiol., 9:935-46.

Ghatak, S. 2020. Strategies for elimination of foodborne pathogens, their influensive detection techniques and drawbacks. In Meat Quality Analysis; Academic Press: Cambridge, 
MA, USA, pp. 267-286.

Kaakoush, N.O., Castano-Rodriguez, N., Mitchell H.M. and Man, S. M. 2015. Global epidemiology of Campylobacter infection. Clin. Microbiol. Rev., 28:687-720.

Mangal, M., Bansal, S., Sharma, S.K. and Gupta, R.K. 2016. Molecular detection of foodborne pathogens: a rapid and accurate answer to food safety. Crit. Rev. Food Sci. Nutr., 56:1568-1584.

Momin, K.M., Milton, A.A.P., Ghatak, S., Thomas, S.C., Priya, G.B., Das, S., Shakuntala, I., Sanjukta RK, Puro K, Sen A. 2020. Development of a novel and rapid polymerase spiral reaction (PSR) assay to detect Salmonella in pork and pork products. Mol. Cell. Probes, 50:101510.

Pan Y, Breidt Jr F. 2007. Enumeration of viable Listeria monocytogenes cells by real-time PCR with propidium monoazide and ethidium monoazide in the presence of dead cells. Appl. Environ. Microbiol., 73:8028-8031.

Priyanka B, Patil RK, Dwarakanath S. 2016. A review on detection methods used for foodborne pathogens. Indian J. Med. Res., 144:327-338.

Singh PK, Jairath G, Ahlawat SS, Pathera A, Singh P. 2016. Biosensor: An emerging safety tool for meat industry. J. Food Sci. Technol., 53:1759- 1765.

Velusamy V, Arshak K, Korostynska O, Oliwa K, Adley C. 2010. An overview of foodborne pathogen detection: in the perspective of biosensors. Biotechnol. Adv., 28:232-254.

Wang Y, Salazar JK. 2016. Culture-independent rapid detection methods for bacterial pathogens and toxins in food matrices. Comp. Rev. Food Sci. Food Safety., 15:183-205. 